\title{
Grafting of Heparin on Blend Membrane of Citric Acid Crosslinked Chitosan/Polyethylene Glycol-Poly Vinyl Alcohol (PEG-PVA)
}

\author{
Retno Ariadi Lusiana ${ }^{1, *}$, Ginanjar Argo Pambudi ${ }^{1}$, Fitra Nilla Sari ${ }^{1}$, Didik Setiyo Widodo ${ }^{1}$, \\ Khabibi $^{1}$, and Sri Isdadiyanto ${ }^{2}$ \\ ${ }^{1}$ Department of Chemistry, Faculty of Sciences and Mathematics, Diponegoro University, \\ Jl. Prof. H. Soedarto, S.H., Tembalang, Semarang 50275, Indonesia \\ ${ }^{2}$ Department of Biology, Faculty of Sciences and Mathematics, Diponegoro University, \\ Jl. Prof. H. Soedarto, S.H., Tembalang, Semarang 50275, Indonesia
}

*Corresponding author:

email: retno.lusiana@live.undip.ac.id

Received: November 25, 2017

Accepted: July 17, 2018

DOI: $10.22146 /$ ijc.30861

\begin{abstract}
Heparin, an active sulfate group material, grafted onto blend membrane of citric acid cross-linked chitosan/ (PVA-PEG) to improve the membrane properties. The physical tests show that grafting reaction of citric acid crosslinked chitosan increased the mechanical strength and membrane swelling. The permeability test results, it was found that the grafted chitosan membrane was improved permeability of both urea and creatinine as compared to pure chitosan and chitosan crosslinked membrane. The negative charge of the sulfonate group of heparin increased the number of the active side of the carrier in the membrane, which then correlated to the membrane's permeability process.
\end{abstract}

Keywords: polymer; grafting; heparin; membrane; permeability

\section{- INTRODUCTION}

Natural polymer materials are widely used as an important material for biotechnology and medical applications due to their unique characteristics such as biocompatibility, non-toxicity, and easy regeneration. Chitosan, a natural material which is potential to be developed as a polymer material for biomedicine because of its nontoxic, antimicrobial, biocompatible, and biodegradable properties [1-2]. Chitosan dissolves in dilute acids to form thin membrane films. However, the mechanical strength of the membrane is not satisfied, this can cause problems when used in medical applications. In addition, functional group in chitosan is not effective as bonding interaction provider (active side) for the target compound [3] and positive charge of amine groups caused complement activation when contacted with blood [4].

In order to increase chitosan membrane mechanical strength, blending with other polymers such as polydimethylsiloxane (PDMS), polysulfone (PSF), polyvinylalcohol (PVA), and polyethersulfone (PES) has been conducted [5-7]. PVA blend was widely developed for medical and pharmacy needs because of its high nontoxic, noncarcinogen, and elasticity characteristics [8-9]. According to Amiji [10] and Ming Chien and Ting-Yu [11] membrane permeability towards creatinine and urea increased in proportion to the increasing of hydrophilic groups in the membrane. In addition, active side quantity and type in the surface and pore lane also determined the permeation speed of target compound (urea and creatinine) [12].

Citric acid (CA) and heparin are widely used as anticoagulant. Although CA has low molecular weight, it cannot be directly used as anticoagulant membrane. In the other side, $\mathrm{CA}$ is an electrophilic agent with three reactive $-\mathrm{COOH}$ groups. Crosslinked between chitosan and citric acid will be resulted to carrying compound with -COO- that functions as membrane active side [2]. The greater the active sides on membrane surface facing source media, the greater the targets that will be captured and carried through the membrane. Interconnection between chitosan amine groups and citric acid will 
reduce of positive charge on the compound when it is used as a membrane.

Heparin acts indirectly in coagulant system by binding anti-thrombin which inactivates thrombin. In high dosage, heparin can inhibit the growth of thrombin [13]. Heparin consists of functional groups such as hydroxyl, carboxylic, and sulfonic that work effectively when mobilized into membrane surface [14]. In addition, those groups can function as active sides of permeate catcher provider. Some studies regarding heparin modification have been conducted to increase membrane [15-17]. According to Zhu et al. [17], modification with direct immerse system is relatively effective to create complex bonds between membrane and heparin. Li et al. [16] conducted heparin modification through direct immobilization with the help of carbodiimide (EDC). This system successfully creates covalent bonds between membrane and heparin.

In this study, citric acid crosslinked chitosan [2] has been grafted using heparin to improve the effectiveness of the active side in the permeation process. In order to increase membrane mechanical, blending using PVAPEG was conducted. Membrane permeated ability was evaluated to determine membrane flux speed towards permeate. Thus we expect the compound resulted from this study can be used as a candidate of hemodialysis membrane.

\section{- EXPERIMENTAL SECTION}

\section{Materials}

All reagent and solvent required in this experiment were obtained commercially and were used without purification. The materials used were: chitosan can be obtained from Biotech Surindo, Cirebon, Indonesia, with deacetylation degree of $87 \%$ (Mw $499.000 \mathrm{Da}$ ), PVA (99.7\% Mw 133.000) was gained from Fisher Scientific, PEG (Mw 3800), acetic acid (glacial, 99.6\%), citric acid (CA, AR), water, $\mathrm{H}_{3} \mathrm{PO}_{4}$ (HP, AR), $\mathrm{Na}_{2} \mathrm{HPO}_{4}$ (DHPD, $\mathrm{AR})$, picric acid (PA, AR), creatinine, urea, 4-amino benzaldehyde, HEP (5000 IU/mL, Mw) were purchased from E. Merck.

\section{Instrumentation}

The instrument used for the transport consisted of two cylindrical chambers glass clamped together with the membrane held between them. The effective volume of each chamber was $50 \mathrm{~mL}$ and the effective area of the membrane was $19.6 \mathrm{~cm}^{2}$. Instruments applied for analysis included Fourier Transform Infrared (FTIR) spectrometer (Shimadzu Prestige-21), Thermal Gravimetric Analysis (TGA) (Perkin Elmer) at the temperature range of $30-900{ }^{\circ} \mathrm{C}$, with heating rate of $5{ }^{\circ} \mathrm{C} / \mathrm{min}$ under nitrogen stream, Scanning Electron Microscopy (JSM-6360 LA), analytical balance, UVspectrophotometer (Shimadzu), pH meter (Hanna), tensile strength meter.

\section{Procedure}

\section{Preparation of blend membranes}

The membranes were prepared by using a phase inversion system. The powder form of chitosan $(1.5 \% \mathrm{w} / \mathrm{v})$ and crosslinked chitosan $(1.5 \% \mathrm{w} / \mathrm{v})$ were dissolved into acetic acid $2 \% \mathrm{v} / \mathrm{v}$ stirred for $24 \mathrm{~h}$. Chitosan-citric acid were prepared by the method that have been reported earlier [2]. PVA-PEG were dissolve into warm distillated water to prepare $1.5 \%$ solution. Chitosan solutions $(10 \mathrm{~mL})$, modified chitosan solution $(10 \mathrm{~mL})$ and modified chitosan/PEG-PVA solution $(10 \mathrm{~mL})$ were cast on petri dish and dried in oven at $50-70{ }^{\circ} \mathrm{C}$ to form thin membranes. The formed membrane was neutralized with $0.03 \mathrm{M} \mathrm{NaOH}$. Membrane was removed from the dish and washed with distilled water until it's neutral and dried with air.

\section{Membrane modification with heparin}

CS-CA/PVA-PEG membrane was soaked in acetate buffer $\mathrm{pH} 4$ that contained $10 \mathrm{~mL}$ of Heparin $100 \mathrm{IU}$ for $24 \mathrm{~h}$. Then it was washed three times using phosphate buffer $\mathrm{pH} 7.4$ and dried at room temperature.

\section{Membrane characterization}

Membrane was tested qualitatively, tensile strength, thickness and weight, swelling, FTIR, surface morphology test with Scanning Electron Microscopy (SEM), heat 
stability (TGA/DTG), and pore Brunauer-EmmettTeller (BET) measurement.

\section{Permeability studies}

The permeation process refers to a method that has been carried out by the same author in the earlier publication [2]. Single solution permeated process was conducted by solving metabolite such as urea (500 ppm) or creatinine (15 ppm) in $50 \mathrm{~mL}$ phosphate buffer into source phase and phosphate buffer without metabolite as acceptor phase. Solute permeability which through membrane was measured by spectrophotometer UV-VIS at one hour interval time until $6 \mathrm{~h}$.

Urea concentration was determined by $p$-dimethyl aminobenzaldehyde (DAB) under acidic condition as the complex which wavelength was measured as $425 \mathrm{~nm}$ (Ehrlich's reagent). Creatinine reacted to picric acid$\mathrm{NaOH}$ and formed colored complex that absorbed radiation in $486 \mathrm{~nm}$ wavelength (Jaffe method). Color formation speed was in proportion directly to creatinine concentration in the sample.

The amount permeated was calculated using the equation [18]

Percentage permeated at time

$\mathrm{t}=\frac{\mathrm{C}_{\mathrm{t}}}{\mathrm{C}_{0}} \times 100$

where $C_{t}$ is the concentration of the solute in the receptor cell diffused at time, $C_{0}$ is the initial concentration of the solute in the donor cell and $t$ is the time in hours.

The permeability coefficient of membranes were calculated using equation [18]:

$\mathrm{P}=\ln \left(\frac{2 \mathrm{Ct}_{0}}{\mathrm{C}_{\mathrm{t}}}-1\right) \times \frac{\mathrm{V}_{\mathrm{s}} \mathrm{l}}{2 \mathrm{a}}$

where $\mathrm{P}$ is the permeability coefficient in $\mathrm{cm}^{2} \mathrm{~min}^{-1}, \mathrm{C}_{\mathrm{t}}$ is the concentration of the solute in the receptor cell at time $t(\mathrm{~min}), \mathrm{C}_{0}$ is the initial concentration of the solute

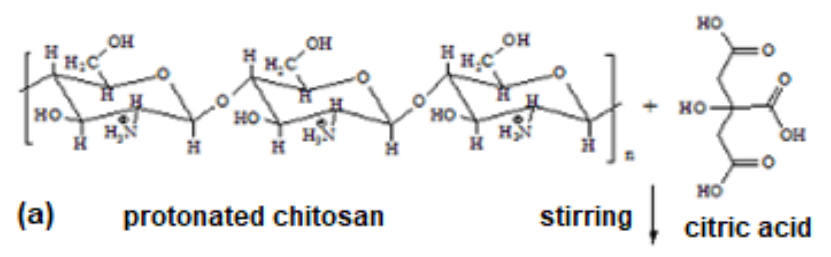

(a)
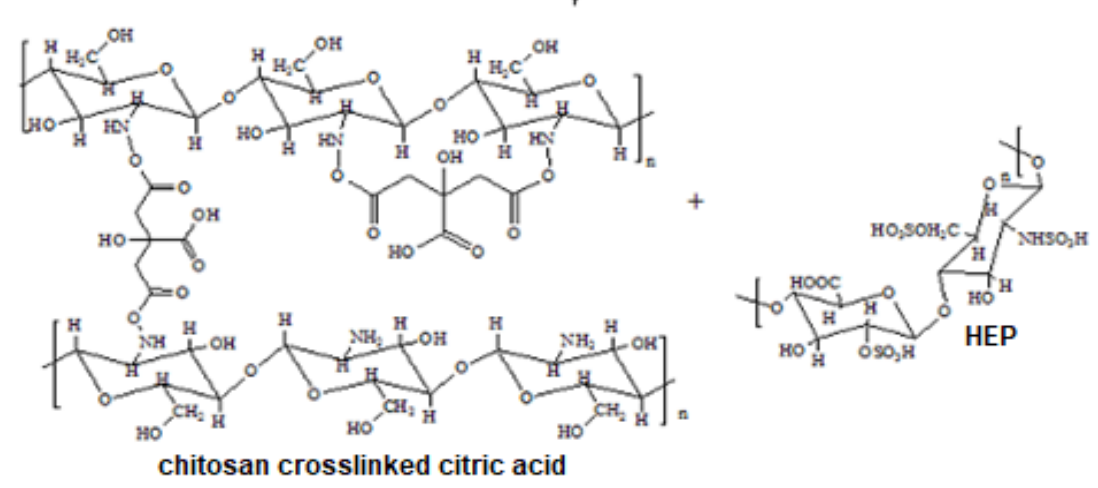

(b)

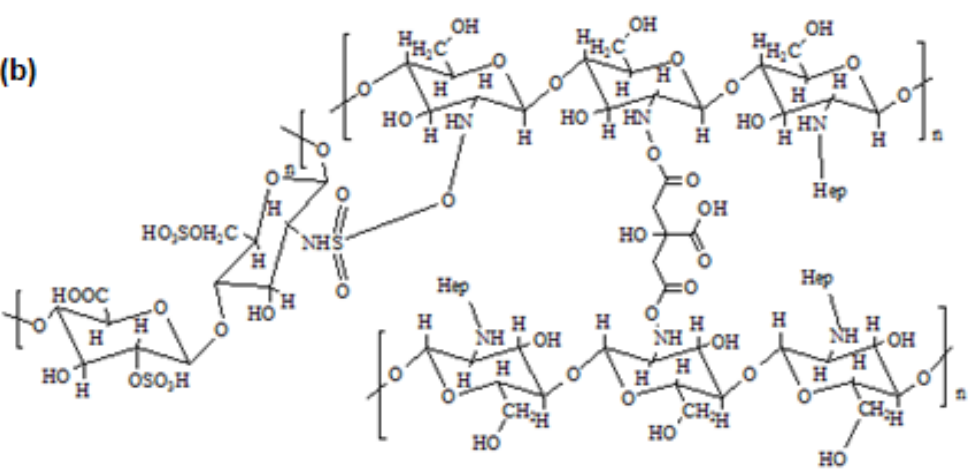

Fig 1. Illustration of the possible reaction sites of (a) chitosan with citric acid, (b) heparin modification [2] 
in the donor cell, 1 is the thickness of membrane in $\mathrm{cm}$, a is the surface area of the membrane in $\mathrm{cm}^{2}$. Permeability coefficient $\mathrm{P}$ is obtained from the slope of the $\left(2 \mathrm{C}_{0} / \mathrm{C}_{\mathrm{t}}-1\right)$ $\times \mathrm{V}_{\mathrm{s}} \mathrm{l} / 2$ a versus time plots.

\section{- RESULTS AND DISCUSSION}

\section{Membrane Characterization}

Membrane thickness is $10 \pm 3 \times 10^{-2} \mathrm{~mm}$, flexible, and clearly transparent.

\section{HEP Grafting Reaction on Surface Membrane}

Reaction of citric acid crosslinked chitosan and heparin modification can be seen in Fig. 1. Citric acid crosslinked towards chitosan reaction was started when $\mathrm{NH}_{2}$ groups were protonated turned into $-\mathrm{NH}_{3}{ }^{+}$resulted from the addition of acid catalyst, this reaction occurred quickly. Therefore $\mathrm{N}$ atom had positive charge and was easily attacked by $-\mathrm{COOH}$ group from citric acid, the reaction in this stage lasted slowly. In addition, ion $\mathrm{H}^{+}$ (catalyst) was resulted which was reformed again in the last stage reaction (Fig. 1(a)).

Grafting reaction mechanism with heparin was started when amino gropus were which was not crosslinked with citric acid, then attacked by $-\mathrm{SO}_{3}$ group from heparin. Either citric acid or heparin could give additional active sites on membrane surface through $\mathrm{COOH}$ group and $-\mathrm{SO}_{3}$. The number of oxygen atom with high electronegativity could create hydrogen with creatinine and urea. In order to see the successfulness of cross-linked reaction and heparin grafted heparin we used spectrophotometer FTIR (Fig. 2).

FTIR analysis result on chitosan membrane showed typical absorption at 3425 and $2877 \mathrm{~cm}^{-1}$ which were $-\mathrm{OH}$ and $-\mathrm{NH}$ group, twin absorption on 1651 and $1597 \mathrm{~cm}^{-1}$ which showed primary amine group $\left(-\mathrm{NH}_{2}\right)$ and showed on the peak of 1381 and $1327 \mathrm{~cm}-1$ which showed -C-O group. FTIR analysis result on citric acid crosslinked chitosan showed wide absorption at 3300$3400 \mathrm{~cm}^{-1}$ that indicated -NH primary group changed into secondary or tertiary and those absorption groups


Fig 2. FTIR spectra of (a) chitosan, (b) citric acid crosslinked chitosan, and (c) heparin modification 
could not be separated from -OH group. The loss of twin absorption into sharp absorption was at $1650 \mathrm{~cm}^{-1}$ which was $\mathrm{C}=\mathrm{O}$ group from carboxylate chain. In the citric acid crosslinked chitosan result, there was no peak which showed $-\mathrm{NH}$ or $-\mathrm{NH}_{2}$ group, but $\mathrm{C}=\mathrm{O}$ group from citric acid showed up [16,19].

There is no fundamental difference from the results of FTIR analysis between chitosan and PVA modified chitosan. Meanwhile FTIR analysis result of citric acid crosslinked chitosan grafted with heparin PVA integration, the peak was formed at $3448 \mathrm{~cm}^{-1}$ which were $-\mathrm{OH}$ group, at 1627 and $1481 \mathrm{~cm}^{-1}$ showed $\mathrm{C}=\mathrm{O}$ and $\mathrm{C}-\mathrm{O}$ groups, as well as 894 and $478 \mathrm{~cm}^{-1}$ that were $-\mathrm{SO}_{3}$ group.

\section{Membrane Mechanical Strength: Tensile Strength}

Membrane tensile strength measurement showed membrane's strength and elasticity that can be seen from tensile strength and strain strength measurement in Table 1. Test results revealed that group modification treatment has improved tensile strength of membranes. Chitosan modification using citric acid, PVA and PEG blend turned the membrane became more elastic. This was possible because of intermolecular interaction between chitosan molecular chain and modifier compound therefore it built cavity between molecules. This cavity increased membrane's elasticity and flexibility. PVA/PEG plasticity characteristic made the membrane more elastic. From all of the data in Table 1, it was found that modification towards chitosan, either by cross-linking or blending can increase membrane's elasticity. By increasing membrane mechanical characteristic, it is expected that the material can withstand the pressure when it is used in permeated process.

\section{Swelling Studies}

Swelling is the process of dissolution of a polymer in a defined solvent [20]. Swelling ability is an important characters of the membrane permeation capability. The dialysis transport process in the membrane needs solvent as a medium to carry transported substance. The transport process will be easier if the membrane has a swelling ratio at a certain range.

The hydrophilicity of a polymer are studied with their swelling capacity and contact angle parameters. The water uptake capacity of membranes has been reported by the same author in one of the earlier publications [2]. Crosslink and transplant reactions performed on chitosan, showed an increase in membrane swelling capacity.

From the results shown in Table 1, crosslinking and grafting reaction of chitosan showed an increased swelling capacity of membranes. With the substitution of carboxylic and sulfate groups in the $-\mathrm{NH}_{2}$ and $\mathrm{OH}$ groups in chitosan, made the number of hydrogen bonding with water in the membrane increases; thereby increasing swelling capacity. The swelling percentage was calculated using equation:

Swelling $(\%)=\frac{\text { weight of swollen membrane }}{\text { weight of dry membrane }} \times 100$

\section{Scanning Electron Microscopy (SEM)}

SEM image represent the morphology of membrane surface. In Fig. 3(a) membrane surface morphology from pure chitosan was smooth, without membrane pores. After modified with PVA-PEG, the blend membrane had more pores (Fig. 3(b)). It was in accordance with PEG characteristics that could increase membrane porosity. High and evenly spread porosity in

Table 1. Data of strain strength (F max), tensile strength, and swelling on various membranes

\begin{tabular}{lccc}
\hline Membrane type & Swelling (\%) & Tensile Strength (MPa) & Strain at F Max (\%) \\
\hline Chitosan (CS) & 104 & 1.50 & 16.40 \\
Chitosan-citric acid (CS.CA) & 260 & 2.73 & 11.41 \\
CS.CA/PVA (CS.CA-1) & 275 & 2.79 & 19.72 \\
CS.CA-1/PEG (CS.CA-1/2) & 234 & 2.54 & 11.93 \\
CS.CA-1/2-hep (CS.CA-1/2-Hep) & 205 & 1.90 & 33.61 \\
\hline
\end{tabular}



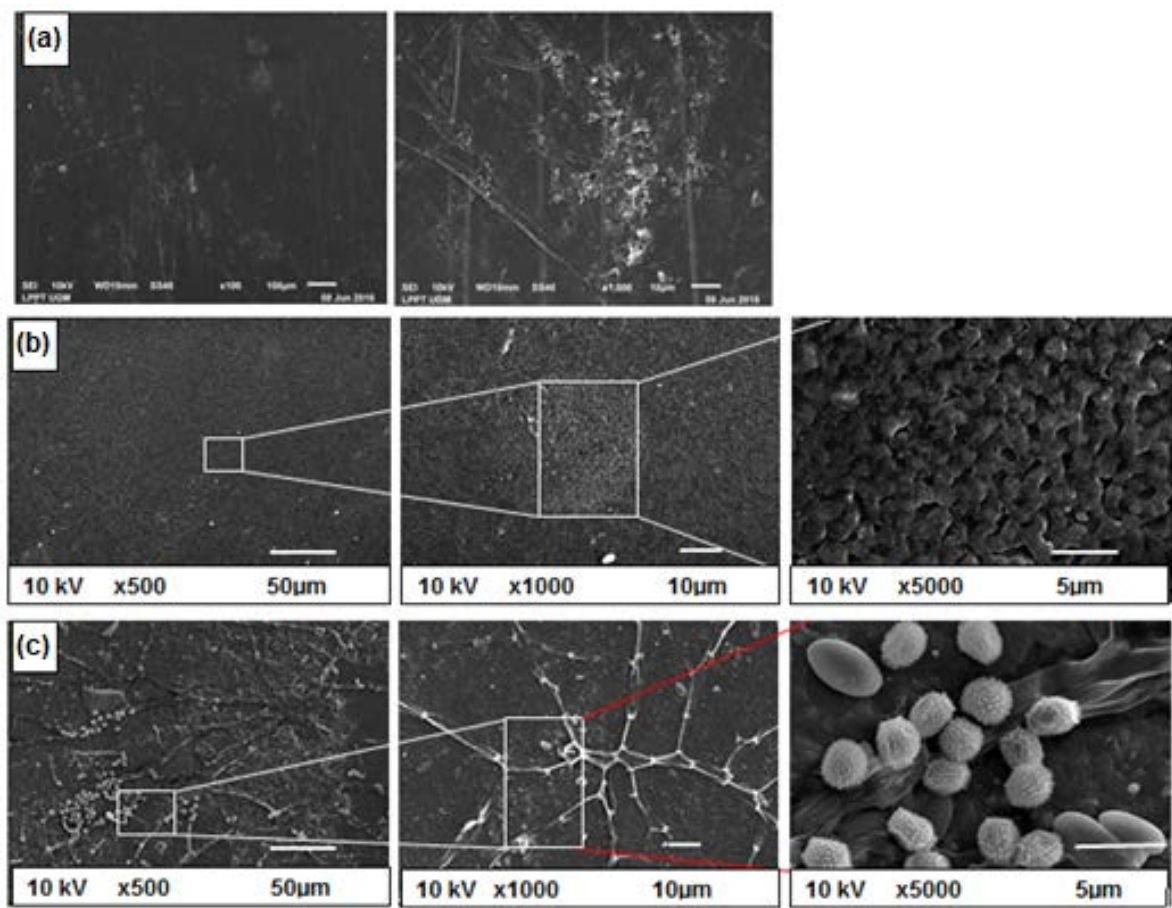

Fig 3. SEM Images from (a) chitosan, (b) modified chitosan blend membrane without heparin, and (c) after mobilized with heparin

all membrane surface was predicted to increase permeated capacity towards permeate. In Fig. 3(c), it showed surface membrane modification towards heparin, causing frayed fibres on some parts of the membrane.

\section{Heat Stability (TGA)}

In order to find out the detailed difference between chitosan and cross-linked chitosan and polymer blend, we prepared thermogram as in Fig. 4. Weight reduction in chitosan occurred in three temperature ranges. The first process was endothermic at approximately $30-100{ }^{\circ} \mathrm{C}$ and weight decrease was $18 \%$. The second weight decrease was at range $120-180$ and $190-320^{\circ} \mathrm{C}$, and weight decrease was until $80 \%$.

Thermogravimetric of citric acid crosslinked chitosan membrane was conducted at temperature range of $30-900{ }^{\circ} \mathrm{C}$, at heating rate of $5{ }^{\circ} \mathrm{C} / \mathrm{min}$. From the thermogram of citric acid crosslinked chitosan, it showed that three levels of compound weight reduction were started from $30-520^{\circ} \mathrm{C}$. The first reduction was related to water evaporation at outer surface of the sample and gases which were easily evaporated like $\mathrm{CO}, \mathrm{CO}_{2}$ generated from $-\mathrm{COOH}$ polymer groups termination. Water evaporation

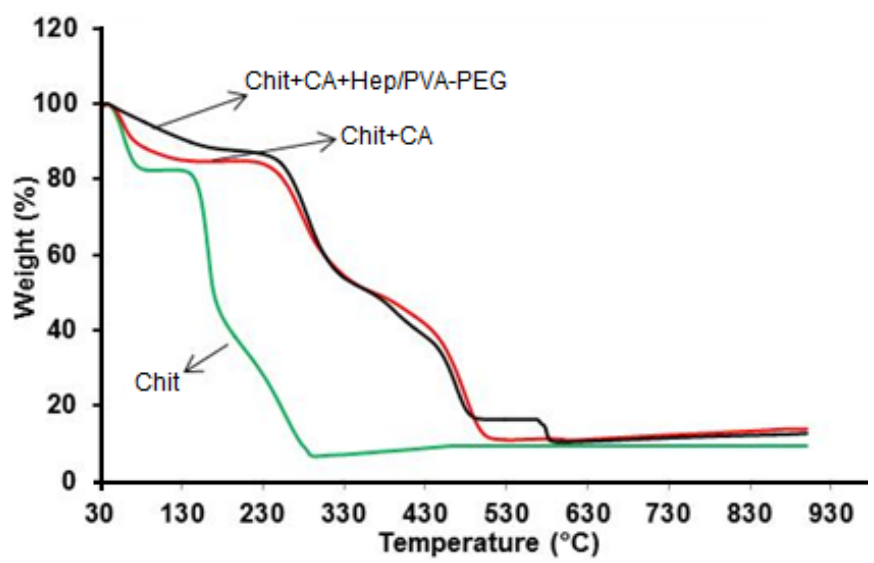

Fig 4. Thermogram TGA of membranes

was associated with compound's hydroxyl content. In this level the reduction of weight was only $10 \%$ at temperature range of $30-100{ }^{\circ} \mathrm{C}$. The second weight reduction was an endothermal process which was related to compound depolymerization into polymer with less molecular weight and the monomers. This stage occurred at temperature range of $200-350{ }^{\circ} \mathrm{C}$ and lost its water as much as $40 \%$. Absorbed water inside the compound was also evaporated at this temperature. The last weight reduction was exothermic at temperature 
range of $350-520^{\circ} \mathrm{C}$ with loss reduction reached $40 \%$. At this temperature range further decomposition occurred towards polymer and opening of glucopyranose ring into smaller elements. Cross-linked group in chitosan base molecule causes compound's thermal stability temperature rose. This looks like similar to the thermogram reported by Fahmi and Fouda [10].

Thermogram from polymer blend membrane was nearly identical with cross-linked membrane thermogram. There were three temperature reductions started at $30{ }^{\circ} \mathrm{C}$, but the last stage showed that after blending with polymer, cross-linked membrane's thermal resistance increased until $600^{\circ} \mathrm{C}$.

\section{Permeation Study}

Hemodialysis membrane strength lies in its ability to pass small molecular weight compounds (urea and creatinine) and prevent larger molecules from flowing out at the same time.

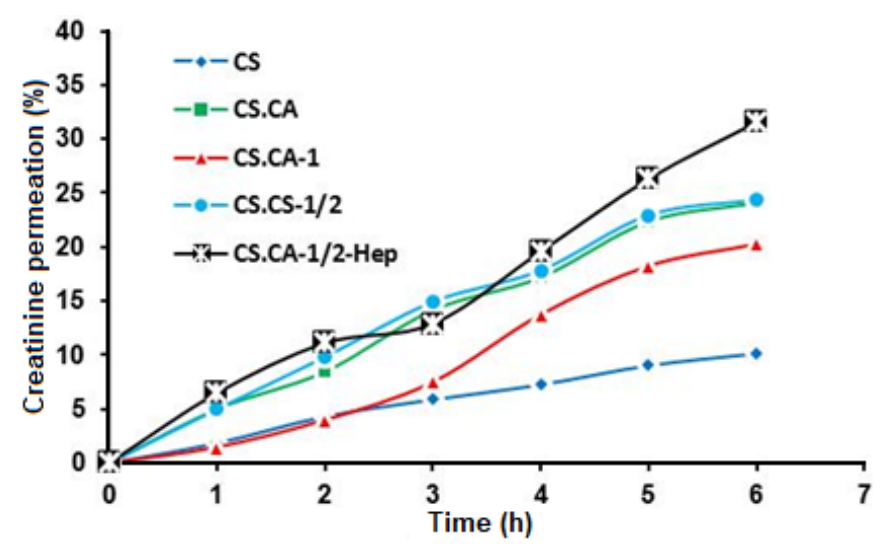

Fig 5. Creatinine permeation through of the membranes
The percentage of urea and creatinine permeated was increasing in proportion to the longer time the membrane contacted with the compound solvent. Membrane ability to pass certain compounds is caused by its ability to swell in an appropriate solvent with certain value to be permeated media of target compound [21].

From Fig. 5 and 6, that are clearly showed that modified chitosan membrane gives higher creatinine and urea permeation percentage compared to pure chitosan membrane. Permeated speed was fast from the initial process up to the $6^{\text {th }}$ hour, and standard point was not reached yet. Permeation significant difference compared with chitosan showed that group modification function through cross-linked reaction with citric acid could add more active sides of membrane material. Modification using PV-PEG did not increase the membrane permeation capability, but only increased the mechanical strength of the membrane. Fuctional group

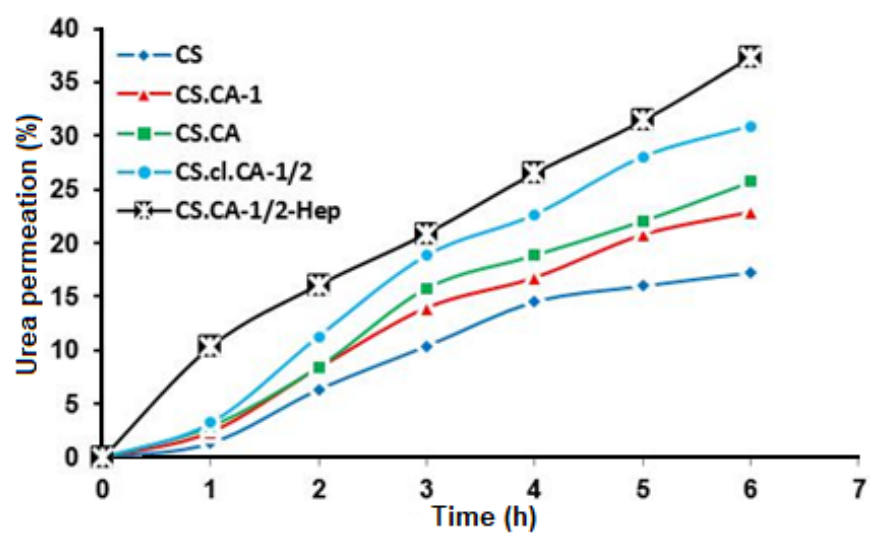

Fig 6. Urea permeation through of the membranes

Table 2. Permeation coefficient of the solutes through membranes

\begin{tabular}{lcccc}
\hline Film & $\begin{array}{c}\text { Thickness } \\
\left(\mathrm{x} 10^{-4} \mathrm{~cm}\right)\end{array}$ & Solute & $\begin{array}{c}\left(\mathrm{P} \times 10^{-3} \mathrm{~cm}^{2}\right. \\
\left.\mathrm{min}^{-1}\right)\end{array}$ & $\mathrm{Kd}$ \\
\hline CS & 12 & & 1.21 & 0.12 \\
CS.CA & 26 & Creatinine & 1.22 & 0.45 \\
CS.CA $1 / 2$ & 24 & & 1.19 & 0.47 \\
CS-CA $1 / 2 \mathrm{Hep}$ & 8 & & 1.26 & 0.84 \\
\hline CS & 12 & & 1.43 & 0.32 \\
CS.CA & 26 & Urea & 1.30 & 0.39 \\
CS.CA $1 / 2$ & 24 & & 1.32 & 0.49 \\
CS.CA $1 / 2 \mathrm{Hep}$ & 8 & & 0.99 & 1.14 \\
\hline
\end{tabular}




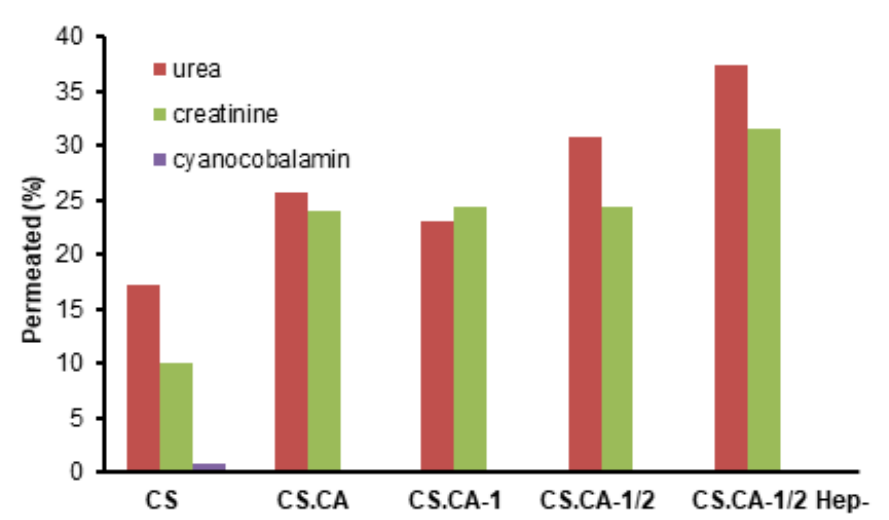

Fig 7. Comparison of permeability percentage of the membranes

modification on membrane surface using heparin $\left(-\mathrm{SO}_{3}\right)$ was able to increase creatinine permeated percentage. It indicated that there were additional active on membrane surface. The increasing number of active site in chitosan because the functional group modification process implements the increased permeation capability by the membrane.

From all of the permeated processes that had been done, the result was consistent which showed urea permeated percentage was higher than creatinine permeated percentage. Urea has molecular weight 60 $\mathrm{g} / \mathrm{mol}$, atomic radius $1.8 \AA$, creatinine $113 \mathrm{~g} / \mathrm{mol}$, molecular size $3 \AA$.

It is generally showed that permeated mechanism by modified chitosan membrane was better than permeated mechanism by pure chitosan membrane for urea and creatinine. Based on the data in Fig. 7 shows that modifications to chitosan can improve membrane permeability. Therefore, we have seen that the permeability of small molecular weight solutes such as creatinine and urea are higher in the chitosan-citric acidheparin film than pure chitosan film. The permeability coefficient of all the membranes calculated as per eq. (2) is given in Table 2 . The urea permeability value of all membranes is much higher than creatinine permeability. On the basis of increased distribution coefficient values, both on urea and creatinine indicate that the membrane is effective enough to pass the metabolite from the source phase to the acceptor phase.

\section{- CONCLUSION}

The main purpose of this study is to find material with high permeated ability. We have demonstrated it by creating material from modifying chitosan through cross-linking blending and grafting reaction. In this study we focused on studying compound additional function as the provider of active sides. From the obtained data, it can be concluded that group modification through crosslinking reaction with citric acid and grafting reaction with heparin could increase membrane permeation ability. The entrance of carboxylate group and sulfate on the chitosan base chain added active sides of chitosan membrane.

\section{- ACKNOWLEDGMENTS}

Funded by source of Diponegoro University DIPA PNBP Fund Number: 573-20/UN7.5.1/PG/2016 and Directorate General of Higher Education through 2017 overseas seminar funding.

\section{- REFERENCES}

[1] Mitra, T., Sailakshmi, G., Gnanamani, A., and Mandal, A.B., 2013, Studies of crosslinking of succininc acid with chitosan/collagen, Mater. Res., 16 (4), 755-765.

[2] Lusiana, R.A., Siswanta, D., and Mudasir, 2016, Preparation of citric acid crosslinked chitosan/ poly(vinyl alcohol) blend membranes for creatinine transport, Indones. J. Chem., 16 (2), 144-150.

[3] Wang, Y.X., Robertson J.L., Spillman W.B., and Claus, R.O., 2004, Effect of the chemical structure and the surface properties of polymeric biomaterials on their biocompatibility, Pharm. Res., 21 (8), 1362-1373.

[4] Anitha, A., Rejinol, S., Bumgardner, J.D., Nair, S.V., and Jayakumar, R., 2012, "Approaches for Functional Modification or Cross-linking of Chitosan" in Chitosan-Based Systems for Biopharmaceuticals: Delivery, Targeting and Polymer Therapeutics, Eds., Sarmento, B., and das Neves, J., $1^{\text {st }}$ ed., John Wiley \& Sons, Ltd., 108-124. 
[5] Rutnakornpituk, M., Ngamdee P., and Phinyocheep, P., 2006, Preparation and properties of polydimethyl siloxane-modified chitosan, Carbohydr. Polym., 63 (2), 229-237.

[6] Costa-Júnior, E.S., Barbosa-Stancioli, E.F., Mansur, A.A.P., Vasconcelos, W.L., and Mansur, H.S., 2009, Preparation and characterization of chitosan/ poly(vinyl alcohol) chemically crosslinked blends for biomedical applications, Carbohydr. Polym., 76 (3), 472-481.

[7] Ran, F., Nie, S., Zhao, W., Li, J., Su, B., Sun, S., and Zhao, C., 2011, Biocompatibility of modified polyethersulfone membranes by blending amphiphilic triblock co-polymer of poly(vinyl pyrrolidone)-b-poly(methyl methacrylate)-b-poly (vinyl pyrrolidone), Acta Biomater., 7 (9), 3370-3381.

[8] Badr, I.H.A., Gouda, M., Abdel-Sattar, R., and Sayour, H.E.M., 2014, Reduction of thrombogenicity of PVC-based sodium selective membrane electrodes using heparin-modified chitosan, Carbohydr. Polym., 99, 783-790.

[9] Silitonga, R.S., Widiastuti, N., Jaafar, J., Ismail, A.F., Abidin, M.N.Z., Azelee, E.W., and Naidu, M., 2018, The modification of PVDF membrane via crosslinking with chitosan and glutaraldehyde as the crosslinking agent, Indones. J. Chem., 18 (1), 1-6.

[10] Fahmy, H.M., and Fouda, M.M.G., 2008, Crosslinking of alginic acid/chitosan matrices using polycarboxylic acids and their utilization for sodium diclofenac release, Carbohydr. Polym., 73 (4), 606-611.

[11] Amiji, M.M., 1995, Permeability and blood compatibility properties of chitosan-poly(ethylene oxide) blend membranes for haemodialysis, Biomaterials, 16 (8), 593-599.

[12] Ming-Chien, Y., and Ting-Yu, L., 2003, The permeation of polyacrylonitrile/polyvinylidine fluoride blend membranes, J. Membr. Sci., 226 (1-2), 119-130.
[13] Abdel-Mohsen, A.M., Aly, A.S., Hrdina, R., Montaser, A.S., and Hebeish, A., 2011, Ecosynthesis of PVA/chitosan hydrogels for biomedical application, J. Polym. Environ., 19 (4), 1005-1012.

[14] Roy, A., and Vikram, K., 2012, Anticoagulation In Haemodialysis, JIMSA, 25, 107-109

[15] Ma, L., Baihai, S., Chong, C., Yin, Z., Qin, H., Zhao, J., Sun, S., and Zhao, C., 2014, Toward highly blood compatible hemodialysis membranes via blending with heparin-mimicking polyurethane: Study in vitro and in vivo, J. Membr. Sci., 470, 90-101.

[16] Li, L., Cheng, C., Xiang, T., Tang, M., Zhao, W., Sun, S., and Zhao, C., 2012, Modification of polyether sulfone hemodialysis membrane by blending citric acid grafted polyurethane and its anticoagulant activity, J. Membr. Sci., 405-406, 261274.

[17] Zhu, A.P., Ming, Z., and Jian, S., 2005, Blood compatibility of chitosan/heparin complex surface modified ePTFE vascular graft, Appl. Surf. Sci., 241 (3-4), 485-492.

[18] Radhakumary, C., Nair, P.D., Nair, C.P.R., and Mathew, S., 2012, Chitosan-graft-poly(vinyl acetate) for hemodialysis application, J. Appl. Polym. Sci., 125 (3), 2022-2033.

[19] Bagheri, M., Younesi, H., Hajati, S., and Borghei, S.M., 2015, Application of chitosan-citric acid nanoparticles for removing chromium(VI), Int. J. Biol. Macromol., 80, 431-444.

[20] Izák, P., Hovorka, S., Bartovský, T., Bartovská, L., and Crespo, J.G., 2007, Swelling of polymeric membranes in room temperature ionic liquids, $J$. Membr. Sci., 296 (1-2), 131-138.

[21] Peppas, N.A., and Reinhart, C.T., 1983, Solute diffusion in swollen membranes. Part I. A new theory, J. Membr. Sci., 15 (1983) 275-287. 